\title{
Noise Source Identification Applied in Electric Power Industry Using Microphone Arrays
}

\author{
Pengxiao Teng, Rilin Chen, Yichun Yang \\ Key Laboratory of Noise and Vibration Research, Chinese Academy of Sciences, Beijing, China \\ Email: px.teng@mail.ioa.ac.cn
}

Received 2013

\begin{abstract}
The noise source identification is an important issue in noise reduction and condition monitoring(CM) for machines insite using microphone arrays. In this paper, we propose a new approach to optimize array configuration based on particles swarm optimization algorithm in order to improve noise source identification and condition monitoring performance. Two distinct optimized array configurations are designed under the certain conditions. Furthermore, an acoustic imaging equipment is developed to carry out experiments on transformer substation equipment and wind turbine generator, which demonstrate that the acoustic imaging system allows a high resolution in identifying main noise sources for noise reduction and abnormal noise sources for condition monitoring.
\end{abstract}

Keywords: Noise Source Identification; Condition Monitoring; Noise Reduction; Microphone Array; Particle Swarm Optimization

\section{Introduction}

As noise reduction and condition monitoring has gained in importance to modern industries, noise source identification has become the focus of a wide variety of research approaches in recent years. In the application of noise reduction on transformer substation or electric equipments, noise source identification is a prerequisite, which facilitates to find out main noise sources from mixed noise field. In addition, the desired noise source can be extracted among mixed sources and acoustic characteristics can be analyzed to find solutions to noise control. In comparison with the mainstream technique of vibration based monitoring, acoustic CMhas the potential to become a generic approach because it has a number of unique features such as generality of acoustic signals in the majority of machines, the richness of information included in acoustic signals and simplicity in sensor placement and hence in CM practice.

Although there has been considerable progress in single-channel acoustic CM in recent years[1,2], acoustic signals are, however, often adversely influenced by their measurement environment and by the range of different acoustic sources within a typical monitoring location. This can make it very difficult to extract useful information for condition monitoring purposes. Recent advances in microphone array technology offer great potential to overcome this problem. Utilizing microphone array technology, an acoustic camera has been invented to vi- sualize sound field which allows to identify main emitting sources. The performance of visualization or localization is significantly affected by the microphone array configuration[3]. The microphone array configuration design is the most crucial parameter to affect localization performance characterized by the array beampattern. It is reasonable to optimize array configuration to form the desirable beampattern with narrow mainlobe width (MLW) and low sidelobe level (SLL) [4] which is in agreement with high spatial resolution and strong capability of interference rejection, respectively.

In array configuration optimization, heuristic methods like genetic algorithm (GA) and particle swarm optimization (PSO)[5,6] are employed due to nonlinear objective functions and constraint conditions. Although GA method performs well in search of solutions in the global space, it is inferior to PSO in finding precise optimal solutions. Particle swarm optimization was initially proposed by Kenney and Eberhart in studying social behavior of bird flocking and fish schooling in their search of food. Particle swarm optimization iteratively updates parameters to converge according to the best individual solution and the best swarm solution. Therefore, it is intuitive for array configuration optimization, and furthermore PSO method is much easier to implement. In this paper, we propose a modified PSO method to optimize an array configuration, which alternatively investigates MLW and SLL. 
This paper is organize as follows. In Sect.2, we give the array configuration model. A modified PSO method is proposed to optimize two distinct array configuration in Sect.3. We develop an acoustic imaging system and carry out experiments in Sect.4 and the conclusion are drawn in Sect.5.

\section{Array Model}

As is known that the noise source identification is highly related to the array beampattern, the beampattern formula is given for the designed array, and then an modified PSO method is proposed to optimize array configuration both according to MLW and SLL in this section.

\subsection{Planar Array Model}

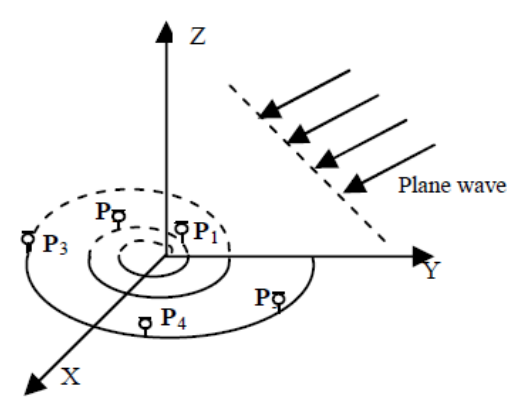

Figure 1. Planar array model

Spiral arrays are widely used due to the merit of low sidelobe. We optimize an array configuration based on spiral structure. Assume that a source signal propagates from the direction $(\theta, \varphi)$ and the coordinate of microphone $p_{m}$ is $\left[r_{m} \cos \phi_{m}, r_{m} \sin \phi_{m}\right]$. The time delay of $m$ th microphone is represented as:

$$
\tau(m)=\frac{r_{m}}{c}\left(\cos \phi_{m} \sin \theta \cos \varphi+\sin \phi_{m} \sin \theta \sin \varphi\right)
$$

Using Eqation (1) , one may obtain the beampattern as:

$$
B(\theta, \varphi)=\sum_{m=1}^{M} w_{m} \exp \left[j \frac{\omega r_{m}}{c}\left(\begin{array}{l}
\cos \phi_{m} \sin \theta \cos \varphi \\
+\sin \phi_{m} \sin \theta \sin \varphi
\end{array}\right)\right]
$$

machine acoustically in a reverberant industrial plant, we design a 3D microphone array with 8 arms at which 8 microphones are installed. An eight-arm 3D microphone array is illustrated in Figure 2. The angle between the $l$ th arm and $z$ axis is denoted as $\vartheta_{l},(l=1,2, \cdots, L)$ and the angle between the projection of the lth arm on the xoy plane and $x$ axis is $\phi_{l},(l=1,2, \cdots, L)$. The time delay of $m_{l}$ th microphone at $l$ th arm is represented by Eqation (3), where $r_{l m_{l}},\left(l=1,2, \cdots, L ; m_{l}=1,2, \cdots, M_{l}\right)$ is defined as the distance from the $m_{l}$ th microphone to the coordinate origin. $\mathrm{M}_{l}$ is the number of microphone at $l$ th arm and $c$ is sound velocity. Using Eqation (3), one may obtain the beampattern by Euqation (4), where $\omega$ is the signal frequency and $w_{l m_{l}}$ is the weight. Without loss of generality, all weights are set to one.

The MLW is defined as $\Theta$ which is the angular interval of the first pair nulls of $|B(\theta, \varphi)|$ for a given $\varphi$ and the sidelobe level is represented as:

$$
\mathrm{SSL}=20 \log _{10} \frac{\left|B_{\notin \Theta}(\theta, \varphi)\right|}{|B(\theta, \varphi)|_{\max }}
$$

where $\notin \Theta$ means sidelobe which is the angular interval outside the mainlobe. Based on the narrow MLW and low SLL criterion, an array configuration can be optimized by

$$
\begin{array}{ll}
\min & \{\mathrm{MLW}, \max \{\mathrm{SLL}\}\} \\
\text { s.t. } & r_{l 1_{l}}=r_{\min }, \quad r_{l M_{l}}=r_{\max }, \\
& r_{l m_{l}}+r_{0} \leq r_{l\left(m_{l}+1\right)} \leq r_{\max }-\left(M_{l}-m_{l}-1\right) r_{0}, \\
& 1 \leq l \leq L, 1 \leq m_{l} \leq M_{l}, r_{0}>0 .
\end{array}
$$

where $r_{l 1_{l}}=r_{\min }$ and $r_{l M_{l}}=r_{\max }$, with $\left[r_{\min }, r_{\max }\right]$ being the distance interval within which microphones are deployed. We also constrain the distance between two adjacent microphones is larger than $r_{0}$. In this paper, we employ PSO method to implement the optimization.

\subsection{D Array Model}

Considering practical applications in imaging large

$$
\begin{gathered}
\tau\left(l, m_{l}\right)=\frac{r_{l m_{l}}}{c}\left(\sin \vartheta_{l} \cos \phi_{l} \sin \theta \cos \varphi+\sin \vartheta_{l} \sin \phi_{l} \sin \theta \sin \varphi+\cos \vartheta_{l} \cos \theta\right) \\
B(\theta, \varphi)=\sum_{l=1}^{L} \sum_{m_{l}=1}^{M_{l}} w_{l m_{l}} \exp \left[j \frac{\omega r_{l m_{l}}}{c}\left(\sin \vartheta_{l} \cos \phi_{l} \sin \theta \cos \varphi+\sin \vartheta_{l} \sin \phi_{l} \sin \theta \sin \varphi+\cos \vartheta_{l} \cos \theta\right)\right]
\end{gathered}
$$




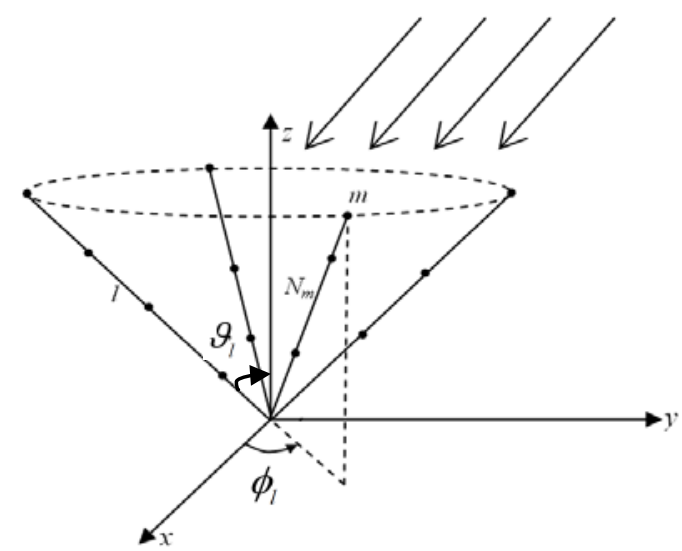

Figure 2. 3D array model

\section{Modified Particle Swarm Optimization}

The aim of employing PSO method to implement equation (6) is to exploit possible microphone positions in order to find a set of optimal position which obtains both narrow MLW and low SLL. Consider a swarm with $N$ particles, each of which represents an array configuration.

$$
\mathbf{X}=\left[\boldsymbol{x}_{1}, \boldsymbol{x}_{2}, \cdots \boldsymbol{x}_{n} \cdots \boldsymbol{x}_{N}\right]^{\mathrm{T}}
$$

where each particle can be denoted as

$$
\boldsymbol{x}_{n}=\left[x_{n 1}, x_{n 2}, \cdots x_{n d}, \cdots x_{n D}\right]
$$

where $D$ is the dimension of optimization. We represent the best individual solution for each particle in the iteration process as:

$$
\mathbf{P}=\left[\boldsymbol{p}_{1}, \boldsymbol{p}_{2}, \cdots \boldsymbol{p}_{n} \cdots \boldsymbol{p}_{N}\right]^{\mathrm{T}}
$$

where $\boldsymbol{p}_{n}=\left[p_{n 1}, p_{n 2}, \cdots p_{n d}, \cdots p_{n D}\right]$ is the best individual solution for the $n$th particle, and the best swarm solution is represented as:

$$
\boldsymbol{g}=\left[g_{1}, g_{2}, \cdots g_{d}, \cdots g_{D}\right]
$$

With Equation(7)through (10), the particles are updated according to the following equations:

$$
\begin{gathered}
v_{n d}^{t}=w \cdot v_{n d}^{t-1}+c_{1} r_{1}\left(p_{n d}-x_{n d}^{t-1}\right)+c_{2} r_{2}\left(g_{d}-x_{n d}^{t-1}\right) \\
x_{n d}^{t}=x_{n d}^{t-1}+v_{n d}^{t}
\end{gathered}
$$

where $C_{1}$ and $C_{2}$ are two positive constants (typically $c_{1}=c_{2}=2$ ), $r_{1}$ and $r_{2}$ are two random variables, and $w$ is inertia weight. The $t$ and $t-1$ represent newly updated variable and previous one, respectively. From Equation(11), we can see that the new velocity is determined by three terms. The first term represents how much the previous velocity is kept. The second term related to the distance between the best individual solution and its current one allows each particle to approach closely to best individual solution. Last term related to the distance between the best global swarm solution and its current one allows each particle to approach closely to best swarm solution. A large inertia weight $w$ tends to explore global area while a small one tends to search local area. Shi[7]suggested a way to determine the inertia weight written in Equation(12) to make a balance in exploring global and local area .

$$
w_{t}=w_{\max }-\frac{w_{\max }-w_{\min }}{T} t
$$

where $w_{\max }$ and $w_{\min }$ are maximum and minimum weight respectively, and $T$ is the total iteration number, $t$ is current iteration index.

We propose a modified PSO method to optimize array configuration based on MLW and SLL in an alternate way. By establishing a upper limit $\zeta(\theta, \varphi)$ for SLL and minimizing mainlobe width, Equation(4) can be rewritten as

$$
\min \{\mathrm{MLW}\}
$$

$$
\begin{array}{ll}
\text { s.t. } & \max \{\operatorname{SLL}\} \leq \zeta(\theta, \varphi) \\
& r_{l 1_{l}}=r_{\min }, \quad r_{l M_{l}}=r_{\max }, \\
& r_{l m_{l}}+r_{0} \leq r_{l\left(m_{l}+1\right)} \leq r_{\max }-\left(M_{l}-m_{l}-1\right) r_{0}, \\
& 1 \leq l \leq L, 1 \leq m_{l} \leq M_{l}, r_{0}>0 .
\end{array}
$$

In order to implement optimization, a fitness value is designed to evaluate the updated particles.

$$
F=\int_{\theta_{0}}^{\theta_{l i m}} \int_{-\pi}^{\pi}\left(B_{\notin \Theta}(\theta, \varphi)-\xi(\theta, \varphi)\right) \delta(\theta, \varphi) d \theta d \varphi
$$

where the integral lower limit $\theta_{0}$ is the first null of $B(\theta, \varphi)$ outside the mainlobe and the integral upper limit $\theta_{\text {lim }}$ is the angle boundary limit. $\left[\theta_{0}, \theta_{\text {lim }}\right]$ is angle volume where we pay more attention to sidelobe. In Equation(14), $\delta(\theta, \varphi)$ is defined as:

$$
\delta(\theta, \varphi)=\left\{\begin{array}{l}
1, B_{\notin \Theta}(\theta, \varphi)>\xi(\theta, \varphi) \\
0, B_{\notin \Theta}(\theta, \varphi) \leq \xi(\theta, \varphi)
\end{array}\right.
$$

As a result, the modified PSO procedure can be summarized as follows: 
STEP1. Initialize PSO parameters including the number of particle $N$, the optimization dimension $D$, particles $\mathbf{X}$, best individual solution $\mathbf{P}$, and best global solution $\boldsymbol{g}$, inertia weight $w_{\min }, w_{\max }$. Set a preliminary SLL and MLW $\theta_{0}$.

STEP2. All particles are updated using Equation(9) through (10).

STEP3. Calculate the fitness value using Equation (12) to evaluate new particles, and judge if $\mathbf{P}$ and $\boldsymbol{g}$ are replaced by newly updated particles.

STEP4. Judge if the fitness value is zero. If so, decrease the $\theta_{0}$ in a small amount and go to step2, and then repeat step2 through step 4. Otherwise, go to step5.

STEP5. Judge if the iteration number reaches the maximum $T$. If not, repeat step2 through step4. Otherwise, go to step6.

STEP6. Terminate and obtain the final result $\boldsymbol{g}$.

In order to demonstrate the performance of optimized array, we calculate the beampattern of the optimized array configuration. Figure 3 shows the beampattern of the optimized planar array configuration. It can be seen that the optimized array configuration has low sidelobe which means better performance of noise source identification. In Figure 4, we compare the beampattern of optimized eight-arm 3D array and uniform array which means that the microphones are distributed evenly at each arm.
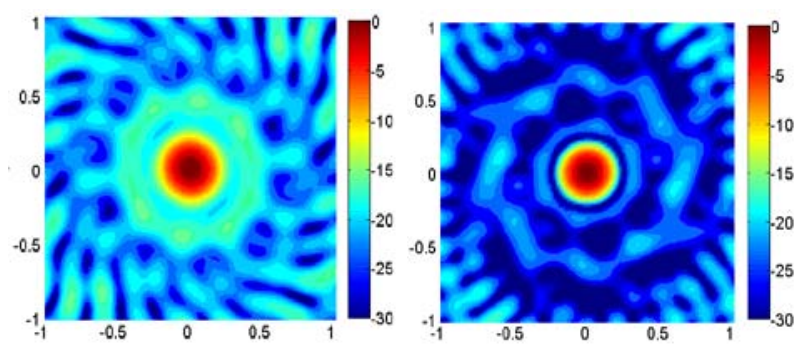

Figure 3. Beampattern comparison of optimized planar spiral array configuration

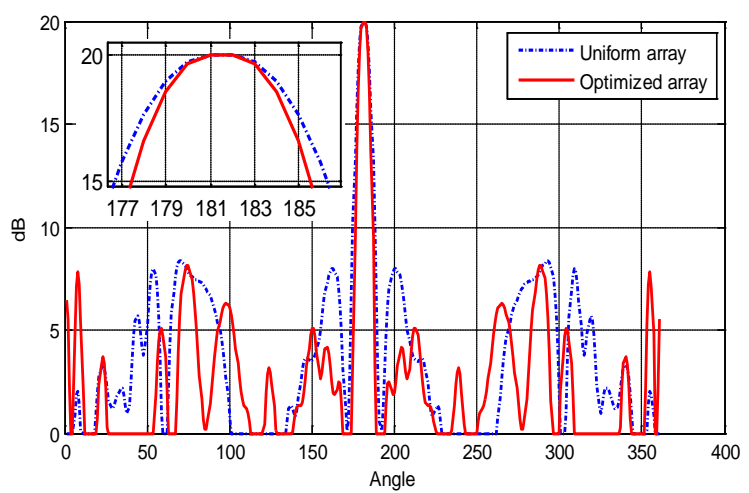

Figure 4. Beampattern comparison of optimized 3D eight-arm array configuration
As shown in Figure 4 inset, the beampattern of optimized array possesses narrower mainlobe and lower sidelobe, which means higher spatial resolution and better noise source identification within focused area.

\section{Experimental results}

An acoustic imaging system is developed based on the two optimized array configuration illustrated in Figure 5. In this section, we employ the acoustic imaging system to identify noise sources and monitor electric power equipments.

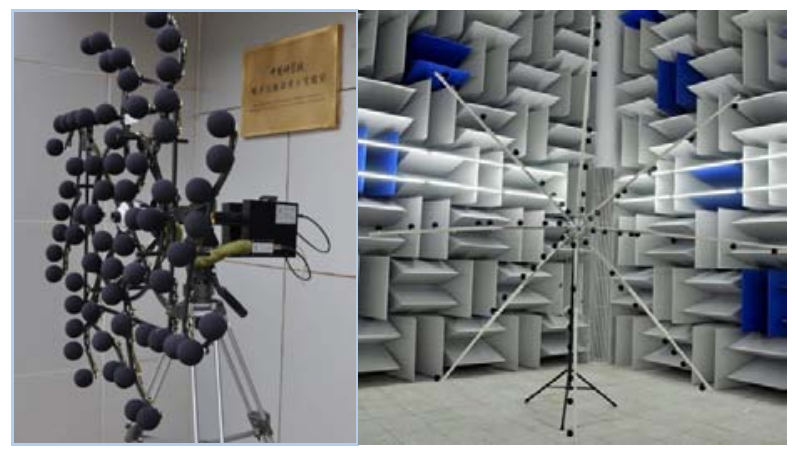

Figure 5. Acoustic imaging system

Figure 6 shows the experiment setup for wind turbine generator and the spectrum of noise sources is displayed in Figure 7.

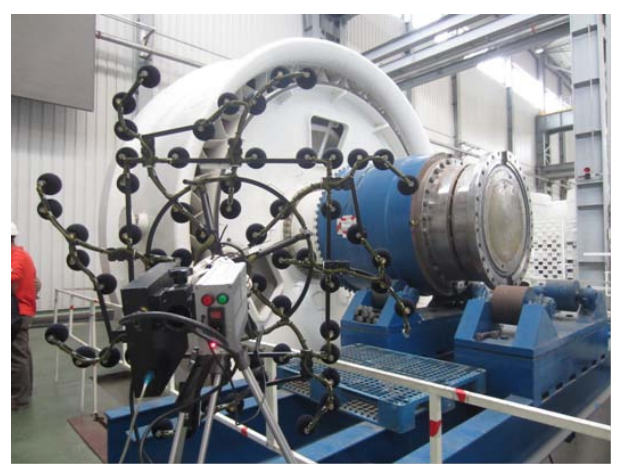

Figure 6. Experiment Setup for wind turbine generator

From Figure 7, it can be observed that there are distinct peaks within the frequency range from $87 \mathrm{~Hz}$ to $119 \mathrm{~Hz}$ and within the frequency range from $180 \mathrm{~Hz}$ to $210 \mathrm{~Hz}$, respectively. The experimental results are illustrated in Figure 8 using the acoustic imaging system. Figure 8(a) shows the noise source emitted from the generator rotor which is known. However, an unexpected noise is finally identified in Figure 8(b), which pinpoint the location where noise comes.

Another example is shown for electric transformer substation equipments from Figure 9 to Figure10. In Fig- 
ure 9, the noise field of substation is visualized to find several main noise sources, which contributes to noise reduction. Figure 10 demonstrates the experimental results of monitoring the operation of a group of fans. Two of them make exceptional strong noise, which means unsteady working state. It is verified by overhaul that the two fans are damaged to some extent.

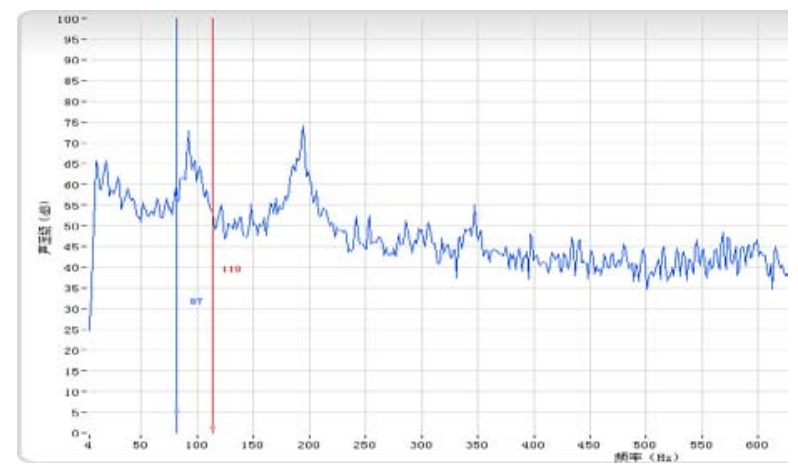

Figure 7. Experiment Setup for wind turbine generator

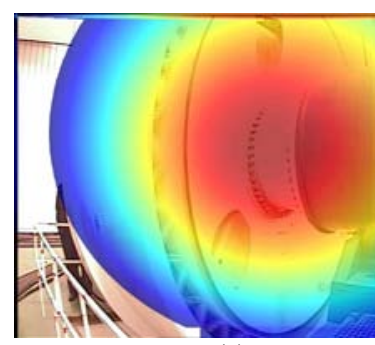

(a)

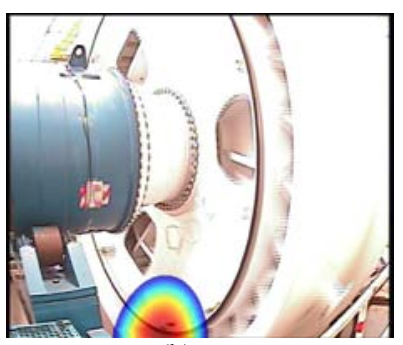

(b)
Figure 8. Noise source identification for wind turbine generator, (a)noise source with frequency range from $87 \mathrm{~Hz}$ to $119 \mathrm{~Hz}$, (b) noise source with frequency range from $180 \mathrm{~Hz}$ to $210 \mathrm{~Hz}$

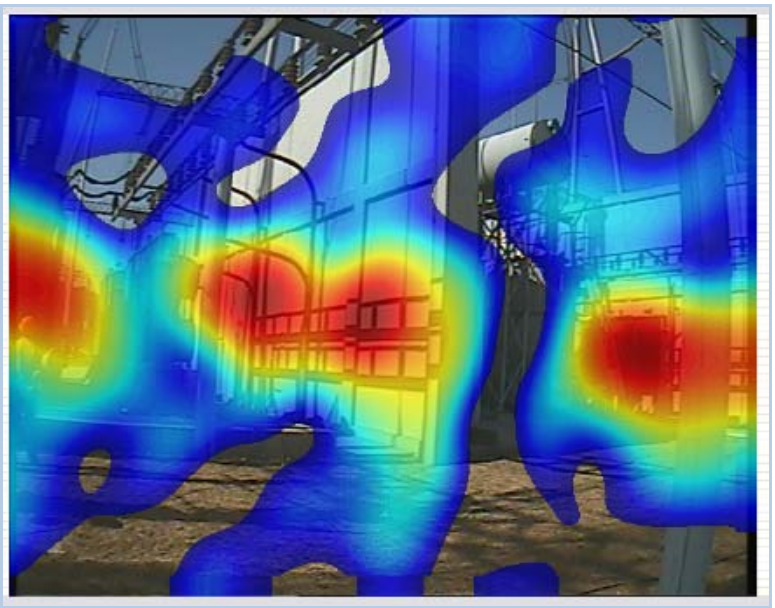

Figure 9. Noise source identification of transformer substation for noise reduction

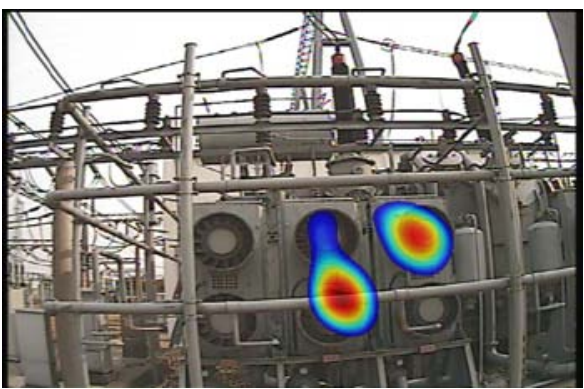

Figure 10. Noise source identification of transformer substation equipment for condition monitoring

\section{Conclusions}

The noise source identification is very important in noise reduction and gaining safe operation of machines. In this paper, array configuration design are discussed in order to improve noise source identification based on particle swarm optimization. Experimental results of wind turbine generator and transformer substation equipment demonstrate the acoustic imaging system can effectively identify main noise sources for noise control and find abnormal noise sources which signify the unsteady state of running machines.

\section{REFERENCES}

[1] A. Albarbar, F. Gu, A. Ball and A. Starr, “On Acoustic Measurement Based Internal Combustion Engines Condition Monitoring," Journal of Non-destructive Testing Institution, Vol.50, 2008, pp. 30-34.

[2] A. Albarbar, F. Gu and A. Ball, "Diesel engine fuel injection monitoring using acoustic measurements and independent component analysis," Measurement, Vol.43, No.10, 2010, pp. 1376-1386.

[3] J. Hald and J. J. Christensen, “A class of optimal broadband phased array geometries designed for easy construction,” Proceedings of Inter-Noise , 2002.

[4] J. Hald and J. J. Christensen, "A novel beamformer array design for noise source location from intermediate measurement distances, ” Proceedings of Inter-Noise ,2002

[5] J. Kennedy, "The PSO: social adaptation of knowledge," in Proc IEEE Int. Conf. on Evolutionary Computation, 1997, pp. 303-308.

[6] D. Mandal and S.Das, "Linear antenna array synthesis using novel particle swarm optimization”, IEEE Symposium on Industrial Electronics and Applications, 2010.

[7] Y. Shi and R. Eberhart, "Parameter selection in particle swarm optimization", Proceedings of the 7th International Conference on Evolutionary Programming, 1447, 1998.

[8] R. Chen and P. Teng, "Spiral array design with particle swarm optimization”, IEEE international conference on signal processing, communications and computing, 2011. 